\title{
Distal Femoral Locking Plates for Tibiotalocalcaneal Fusions in the Charcot Ankle: A retrospective study
}

 \\ Vineet Kamboj, DPM $^{4}$
}

The Foot and Ankle Online Journal 4 (8): 3

This paper presents a retrospective case series with chart and radiographic review of four patients with Charcot neuroarthropathy and associated ankle valgus. All four patients underwent tibiotalocalcaneal (TTC) arthrodesis using a distal femoral locking plate combined with external ring fixation for rigid axial compression. A 12 month follow-up was obtained. All four TTC arthrodeses were performed by the same surgeon (BH) including preoperative and postoperative evaluation and care. Outcomes were deemed successful with evidence of radiographic consolidation across the fusion sites. Outcomes were considered failures in the presence of non-union or amputation. Three patients had satisfactory outcomes with only minor complications. One patient had failure of the procedure with development of osteomyelitis and ultimately had a below knee amputation. Although this was a small review, on average, osseous consolidation was appreciated in 77 days for those patients that had successful outcomes. Larger retrospective or even prospective studies are needed to confirm the use of tibiotalocalcaneal arthrodesis using a distal femoral locking plate and external ring fixation in Charcot arthropathy. This small case series shows promise to the efficacy of distal femoral locking plates for tibiotalocalcaneal fusions.

Key words: Tibiotalocalcaneal fusions, Charcot Ankle, distal femoral locking plates, ankle valgus

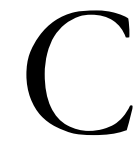

harcot neuroarthropathy when left untreated can progress to combined deformity of the ankle and subtalar joints. The treatment goal in these cases is to produce a stable, plantigrade foot that is braceable.

\footnotetext{
Address correspondence to: Sarah Shogren, DPM, Franciscan Foot and Ankle Institute, $345099^{\text {th }}$ Ave S. Ste 306 Federal Way WA 98003.

Email: SarahShogren@fhshealth.org

${ }^{1-4}$ Franciscan Foot and Ankle Institute, $345099^{\text {th }}$ Ave S. Ste 306 Federal Way WA 98003.
}

When conservative treatments such as bracing and shoe gear modifications fail, tibiotalocalcaneal (TTC) arthrodesis is typically the salvage procedure of choice. Bone quality in Charcot patients is often poor due to osteoclastic activity. The decision on the type of stable fixation to use can often be a challenge.

Ahmad et al., described humeral locking plates to have a high rate of fusion at 94.1\%. ${ }^{1}$ Several forms of stable fixation have been used to achieve TTC arthrodesis, including screws, intramedullary nails, ${ }^{3}$ blade plates, ${ }^{3}$ external fixation, ${ }^{4,6}$ and humeral locking plates, ${ }^{5}$ to name a few. 




According to Pelton and Carvaggi, intramedullary nails are a good method for TTC arthrodesis with $88 \%$ and $92.8 \%$ fusion rates respectively.,

Distal femoral locking plates, like humeral locking plates, are not made specifically for this reconstruction; but are stable with a viable architecture to encompass both ankle and subtalar joints. The locking plate technology allows for a more stable construct in patients with questionable bone quality and combined deformities as in Charcot neuroarthropathy. (Figs. 1A and 1B)

The purpose of this retrospective chart review was to evaluate the efficacy of distal femoral locking plates for tibiotalocalcaneal fusions.

\section{Methods}

A retrospective chart and radiographic review of four patients with Charcot neuroarthropathy with associated ankle valgus was studied. Inclusion criteria for this case series were patients with Charcot neuroarthropathy and a painful deformity at the ankle and subtalar joints. Exclusion criteria included active infections, significant bone loss from trauma and osteonecrosis. All patients failed conservative treatments, including bracing and shoe gear modifications, and all requested definitive treatment. Due to the retrospective review of these patients pain scales were not compared before and after surgery.

All four patients underwent tibiotalocalcaneal arthrodesis using a distal femoral locking plate combined with external ring fixation for rigid axial compression. Follow-up was obtained for up to 12 months. Frames were removed after approximately three months, after which patients began progressive weightbearing in a postoperative boot.

All four TTC arthrodeses were performed by the

Figure 1A and 1B Pre-operative radiograph $(A)$ and patient (B) with combined Charcot ankle valgus and subtalar deformities. same surgeon, including preoperative and postoperative evaluation and care. Outcomes were deemed successful with evidence of radiographic and clinical evidence of consolidation across the fusion sites. Outcomes were considered failures in the presence of non-union or amputation. 




Figures 2A and 2B Surgical resection of the fibula (A) is performed prior to resection of the tibiotalar joint. (B)

\section{Surgical Method}

A linear incision was made adjacent to the course of the fibula. An oblique osteotomy was made at the distal third of the fibula. The fibula was resected and removed from the operative table. The tibiotalar joint was then resected with the sagittal saw. (Figs. 2A and 2B) An ankle arthrotomy was performed medially and the medial malleolar articular surface was resected with a sagittal saw forming a miter at the medial aspect of the tibial surface. (Fig. 3) The subtalar joint was denuded of cartilage using a curette.

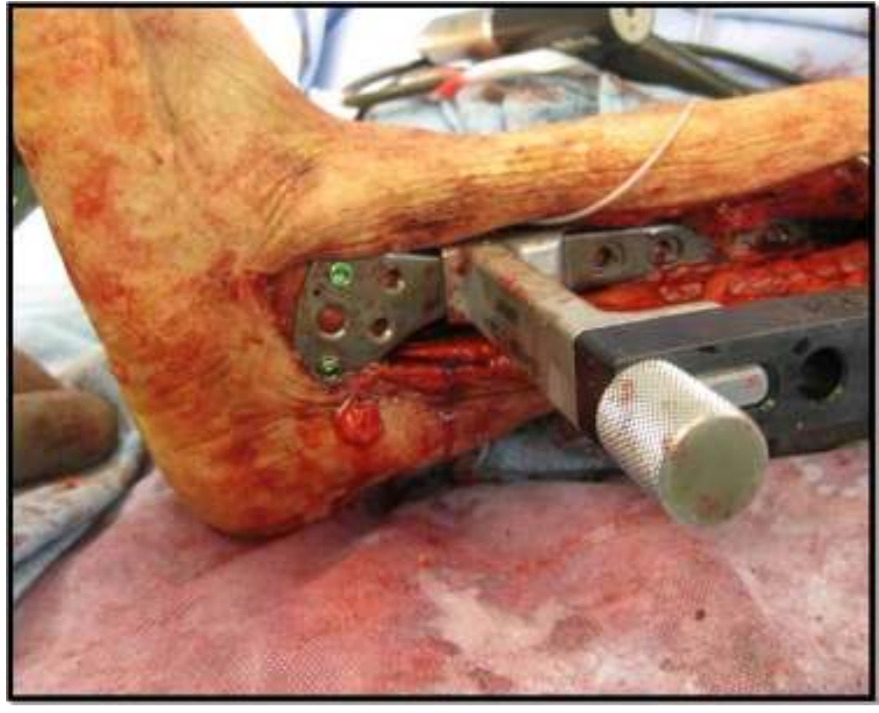

Figure 3 Application of the distal femoral locking plate to stabilize the Charcot ankle is technically simple.

The tibiotalocalcaneal arthrodesis was temporarily fixated with a Steinman pin placed through the plantar aspect of the foot crossing both joints into the tibia. The distal femoral locking plate was applied to the lateral aspect of the tibia, talus and calcaneus. Multipotential cellular bone matrix as well as platelet rich plasma were added to the autogenous bone graft which was placed in the tibiotalar arthrodesis site.

Accurate placement of the plate was verified using intraoperative fluoroscopy, and the Steinman pin was removed from the plantar foot. The circular fixator frame was then applied to the lower extremity with wires and half pins using standard technique. (Fig. 4) Axial compression was applied through the external fixator.

\section{Outcomes}

All four patients remained non-weightbearing for roughly three months in the external fixator. The fixator was then removed and patients remained nonweightbearing for an additional two weeks in a CAM boot. Patients began progressive weightbearing in a post-operative boot to full weightbearing for 3 additional months. 


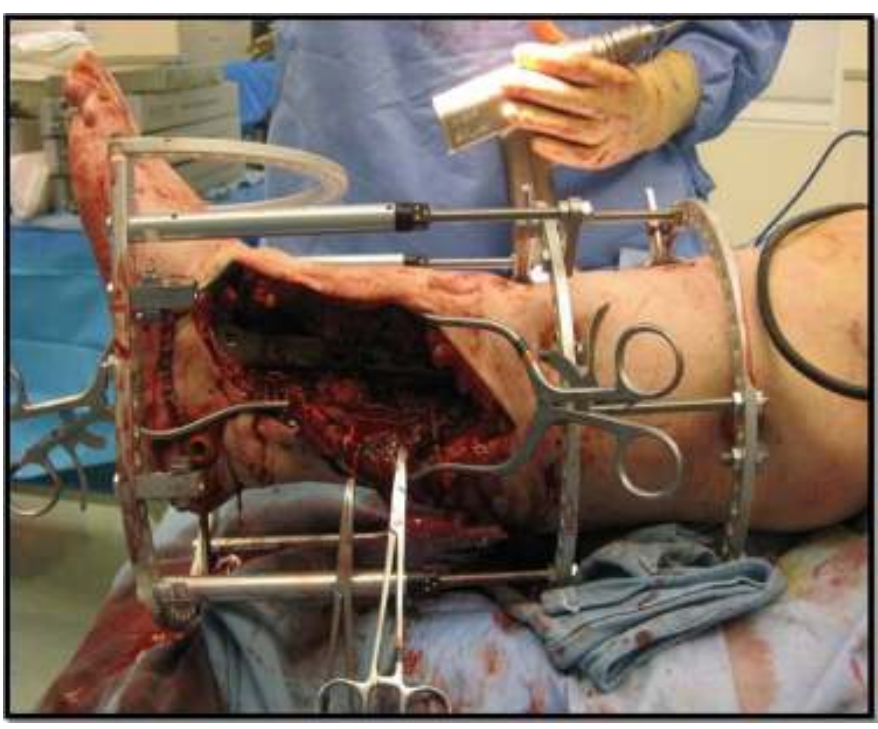

Figure 4: Application of external fixator to aid in axial compression of the Charcot Ankle.

Once radiographic consolidation was seen, patients were progressed to full weightbearing in a CROW (Charcot Restraint Orthotic Walker) boot or similar device. These patients were ambulating with minimal pain at the end of the postoperative recovery period and able to perform their daily living requirements.

Three patients $(75 \%)$ had satisfactory outcomes with only minor complications. Two patients (50\%) required blood transfusion following surgery, and one $(25 \%)$ had mild pin tract infections which responded quickly to oral antibiotics. One patient $(25 \%)$ had failure of the procedure with development of osteomyelitis and ultimately had a below knee amputation. This patient did not have an active infection at the time of surgery however; this patient had a history of prior osteomyelitis from previous procedures and was in renal failure. These preoperative factors ultimately could have contributed to the patients post- operative wound dehiscence and ultimate recurrence of osteomyelitis. On average, osseous consolidation was appreciated in 77 days for those patients that had successful outcomes. (Fig. 5) The patients with successful outcomes were able to ambulate pain free in a CROW boot. Table 1 summarizes the four patient outcomes.

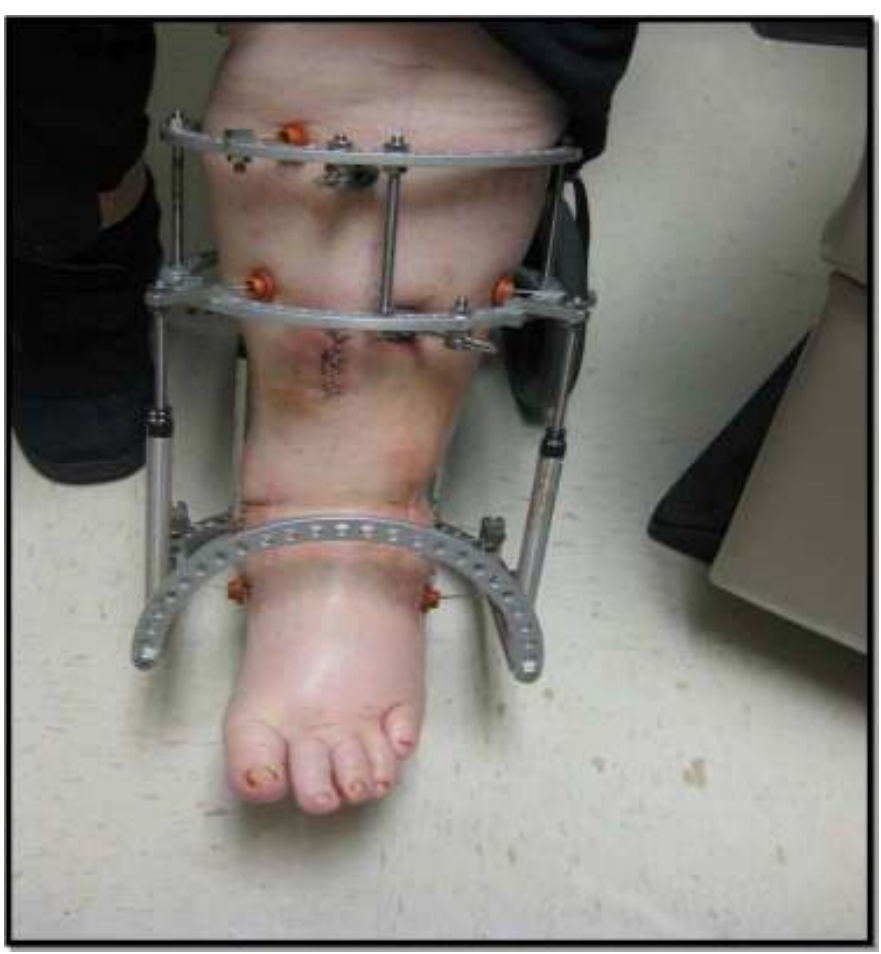

Figure 5 Clinical presentation during 3-month course in external fixators.

\section{Discussion}

Approaching the treatment for a patient with Charcot arthropathy can be very difficult and controversial. These patients generally have multiple comorbidities making healing potential for ulcerations, as well as surgical procedures, more difficult. If ulceration prevention and adequate mobility is achieved using a bracing method this should be done as first line treatment. $^{8}$

Attaining rigid fixation in a tibiotalocalcaneal arthrodeses can be difficult in patients with Charcot neuroarthropathy. Chiodo, et al., in 2003, compared the biomechanical properties of blade-plates and intramedullary rod fixation for TTC arthrodesis, and found the blade-plate to be a more rigid construct. ${ }^{3} \mathrm{It}$ is important to note that while blade plates do give a more rigid construct, they do not offer multiple planes of fixation. Intramedullary rods have been shown to have high fusion rates however they are technically difficult with risk of stress risers, fractures and neurovascular injury. 


\begin{tabular}{|l|c|c|c|c|c|}
\hline \multicolumn{7}{|c|}{ Tibiotalocalcaneal Fusion Patient Outcomes } \\
\hline & $\begin{array}{c}\text { Frame Removal } \\
\text { (days) }\end{array}$ & $\begin{array}{c}\text { Full WB } \\
\text { (days) }\end{array}$ & $\begin{array}{c}\text { Bone } \\
\text { Consolidation } \\
\text { (days) }\end{array}$ & Complications & Outcome \\
\hline Patient\#1 & 70 & 82 & 82 & Required blood transfusion & Satisfactory \\
\hline Patient\#2 & 71 & 121 & 88 & Required blood transfusion & Satisfactory \\
\hline Patient\#3 & 83 & 152 & none & Wound dehiscence, osteomyelitis & Failure \\
\hline Patient\#4 & 84 & 108 & 60 & Pin tract infection & Satisfactory \\
\hline
\end{tabular}

Table 1 Tibiotalocalcaneal fusion outcomes for the four patients included in this retrospective case series.

Pinzure and Kelikian reported 21 ankles with Charcot arthropathy treated using the intramedullary nail. Ninety per cent of these patients went on to fusion. The authors concluded this as an excellent means of obtaining ankle fusion in Charcot patients. In comparison, another form of fixation are locking plates which are less technically demanding and offer fixation in multiple planes. $^{5}$ Fixation in multiple planes is important because it limits rotational forces. Ahmad, et al., proved that using a PHILOS locking plate to achieve TTC arthrodesis does provide bone union and deformity correction. In patients with Charcot neuroarthropathy, greater rigidity is ideal to maintain the correction through the arthrodesis and we believe the locking plate will do this.

The greatest limitation to our case series is a small population size. Further research needs to be done in a prospective manner. This would give the advantage of determining a standardized pre-operative pain score (AOFAS) which is another limiting factor in this retrospective review.

The use of distal femoral locking plates for tibiotalocalcaneal arthrodesis is a viable rigid internal form of fixation. The locking plate technology allows for a stable construct in patients with questionable bone quality. In comparison to other forms of arthrodesis, it has a more rigid construct with better boney apposition and fixation in multiple planes.
In summary, the use of distal femoral locking plates in conjunction with external fixation is an acceptable option to create a plantigrade braceable foot as a limb salvage procedure in Charcot neuropathy patients.

\section{References}

1. Ahmad J, Pour AE, Raikin SM. The modified use of a proximal humeral locking plate for tibiotalocalcaneal arthrodesis. Foot Ankle International 2007 28: 977-983.

2. Carvaggi C. Intramedullary compressive nail fixation for the treatment of severe Charcot deformity of the ankle and rearfoot. J Foot Ankle Surg 2006: 45(1), 20-24.

3. Chiodo CP, Acevedo JI, Sammarco VJ, Parks BG, Boucher HR, Myerson MS, Schon LC. Intramedullary rod fixation compared with blade-plate-and-screw fixation for tibiotalocalcaneal arthrodesis: A biomechanical investigation JBJS 2003 83A: 2425-2428.

4. Colgrove RC, Bruffey JD. Ankle arthrodesis: Combined internal-external fixation. Foot Ankle International 2001 22: 9297.

5. Lowery NJ, Alison JM, Burns PR. Tibialtalocalcaneal arthrodesis with the use of a humeral locking plate. Clinics Podiatric Medicine Surgery. 2006 26: 485-492.

6. Misson JR, Anderson JG, Bohay DR, Weinfeld SB. External fixation techniques for foot and ankle fusions. Foot Ankle Clinics 2004 9: 529-539.

7. Pelton K. Tibiocalcaneal arthrodesis using a dynamically locked retrograde intramedullary nail. Foot Ankle International 2008: 27: 759-763.

8. Pinzure M. Surgical versus accommodative treatment for Charcot arthropathy of the midfoot. Foot Ankle International 2004 25: 545-549.

9. Pinzure MS, Kelikian A. Charcot ankle fusion with a retrograde locked intramedullary nail. Foot Ankle International 1997: 18, 699-704. 